\title{
Distribution of methanogenic archaea in different oral sites amongst subjects with halitosis
}

\author{
Vasanth Ayswarya ${ }^{1, *}, V^{\prime} a m s i$ Lavu ${ }^{2}$, Suresh Ranga Rao ${ }^{3}$ \\ ${ }^{1}$ Senior Lecturer, ${ }^{2}$ Associate Professor, ${ }^{3} \mathrm{HOD}$ (Retired), Dept. of Periodontics, ${ }^{\text {P Priyadarshini Dental College and Hospital, Tamil }}$ \\ Nadu, ${ }^{2,3}$ Sri Ramachandra Dental College \& Hospital, Chennai, Tamil Nadu, India
}

*Corresponding Author: Vasanth Ayswarya

Email: vvayswarya@gmail.com

Received: $1^{\text {st }}$ October, 2018

Accepted: $20^{\text {th }}$ November, 2018

\begin{abstract}
Introduction: Methanogenic archaea are single celled methanogenic anaerobic microorganisms that are potential hydrogen competitors. It serves as a syntrophic partners with other members of the subgingival biofilm community leading to methanogenesis, sulfate reduction and acetogenesis thereby producing hydrogen sulfide, a potent Volatile Sulphur compound to cause halitosis.

Aim: To evaluate the presence of methanogenic archaea in supra gingival plaque, subgingival plaque and tongue coating in subjects with and without oral halitosis.

Materials and Methods: A case control study of 50 subjects with 25 halitosis and 25 healthy subjects were included in the study. Supragingival plaque, subgingival plaque and tongue coating were collected from all the subjects. DNA extraction was done from the samples by Bacterial genome extraction kit. Conventional PCR was done to detect the presence of Methanogenic archaea.

Results: The prevalence of methanogenic archaea in supra gingival plaque, subgingival plaque and tongue coating in halitosis subjects were $16 \%, 60 \%$ and $16 \%$ respectively and in controls without halitosis were $4 \%, 8 \%$ and $0 \%$ respectively. A highly statistical significant difference (p 0.000) was found between subgingival plaque samples of cases and controls.

Conclusion: A significantly higher presence of methanogenic archaea was observed in halitosis subjects than controls, with increased detection in the subgingival plaque thereby fulfilling the association criteria of Socransky's postulates. However, a larger sample size, identification of VSC production and synergistic activity with other microorganisms needs to be established.
\end{abstract}

Keywords: Halitosis, Methanogenic Archaea, Plaque, Tongue coating, Oral malodor.

\section{Introduction}

Archaea are a group of microorganisms that differ fundamentally from eukaryotes and bacteria in several genetic, biochemical and structural features and thrive in extreme environments such as hot springs, salt lakes or submarine volcanic habitats. ${ }^{1}$ The distinction between the domains bacteria and archaea is based mainly on the different types of ribosomal RNA and the chemical nature of the membrane lipids. Archaea possess unique flagellins and ether-linked lipids and lack murine, a peptidoglycan that forms rigid cell wall saccule in almost all bacteria. ${ }^{2}$ Almost $15 \%$ of the proteins encoded by each archaeal genome are unique to archaea. A set of archaeal signature genes support the phylogenetic conclusion that archaea are an anciently diverged major lineage containing a substantial proportion of unique genes. ${ }^{3}$

Archaea are methanogens which produce methane from various substrates such as hydrogen and carbon dioxide, methylated carbon compounds or acetate. ${ }^{4}$ Some archaea can be isolated from oral cavity, human gut and vagina. ${ }^{5}$ In oral cavity, archaea can be isolated from subgingival samples of periodontitis patients, infected root canals and peri-implantitis. ${ }^{6-8}$ Methanobrevibacter is the major genus isolated oral cavity, of which majority were Methanobrevibacter oralis like species. ${ }^{9}$ Viable counts of Methanogenic archaea bacteria and sulfate reducing bacteria are found in oral cavity mostly in supra gingival and subgingival plaque. ${ }^{10}$ The subgingival plaque from periodontitis patients harbors a larger number of total bacteria; the hydrogenotrophic group includes methanogenic archaea and sulfate-reducing bacteria in addition to acetogenic bacteria. Vianna et al found that the latter two groups are absent in healthy control subjects but present in $65 \%$ of periodontitis patients, alone or in combination. ${ }^{11}$

Oral halitosis is a condition in which foul or offensive odour emanates from the oral cavity caused both due to intra-oral or extra-oral reasons. Intraoral halitosis may be indicative of either oral diseases, such as periodontal diseases, or the presence of excessive bacterial reservoirs on the tongue. ${ }^{12}$ Extra-oral halitosis can be due to chronic sinusitis, nasal obstruction, carcinoma of lungs, bronchitis, bronchiectasis, pneumonia, pulmonary abscess, hiatus hernia, hepatic cirrhosis or diabetes mellitus. ${ }^{13}$ However, many epidemiological studies have shown that the $90 \%$ of halitosis cases have its origin in the oral cavity. ${ }^{14}$

The etiology of oral halitosis has been mainly related to the putrefactive activities of Gram-negative anaerobic bacteria, in particular, the bacterial degradation of Sulfur containing amino acids such as methionine, cystine and cysteine into volatile sulfur compounds(VSC); mostly hydrogen sulfide, methyl mercaptan and dimethyl sulfide. ${ }^{15}$ Various studies 
concluded that Gram-negative bacteria are potent producers of VSC. ${ }^{16}$

The human oral cavity contains complex heterogenous microflora. Proteolytic activity of the bacteria causes the production of VSC. Treponema denticola, Porphyromonas gingivalis, Prevotella intermedia, Bacteroides forsythus and Fusobacterium species can produce significant amounts of $\mathrm{H}_{2} \mathrm{~S}$ and methylmercaptan. ${ }^{17}$ Periodontal pathogens have known to be associated with VSC production suggesting increased prevalence of malodor in subjects with periodontitis compared to health.

A study by Zhu correlated Porphyromonas gingivalis in subgingival plaque and spirochetes in tongue to produce VSC which reduced significantly after periodontal therapy. ${ }^{18}$ Another study correlated Porphyromonas gingivalis, Tannerella forsythia, Prevotella intermedia, Prevotella nigrescens, Treponema denticola, species of Veillonella, Actinomyces and Prevotella on plaque and tongue dorsa contributing greatly to VSC production. ${ }^{19,20}$ A study by Tangerman concluded that methyl mercaptan and to a lesser extent hydrogen sulphide were the main contributors to intra-oral halitosis; dimethyl sulphide were the main contributor to extra-oral or blood-borne halitosis. ${ }^{21}$ It was also studied that an increased levels of cadaverine and galactosidase activity in saliva were responsible for VSC. ${ }^{22,23}$

Various bacteria and factors have been studied and associated for causing halitosis, but the etiology is not concluded to one as a causative factor. The micro-niche condition existing in human periodontal pockets is ideal for growth of methanogens, which are strictly anaerobes. ${ }^{24}$ Archaea, a strict methanogen, along with other sulfate reducing bacteria and acetogenic bacteria produces hydrogen sulfide, a potent VSC causing halitosis. Tonzetich reported that VSC are the main component of halitosis and $90 \%$ of it is made of hydrogen sulfide. ${ }^{25}$ Thus, Methanogenic archaea, commonly found in oral cavity, has been proposed to be linked to sulfate reducing bacteria which would be a major contributing factor to oral halitosis. Until date the role of methanogenic archaea to cause oral halitosis has not been evaluated and remains a lacunae of knowledge. This study aims to determine the presence and distribution of methanogenic archaea in different oral sites amongst subjects with oral halitosis.

\section{Materials and Methods}

A total of 50 subjects - 25 with halitosis and 25 without halitosis were included in this study, from the out-patient ward of the department of Periodontics, Sri Ramachandra University, Chennai - 600116. The study was performed after obtaining approval from Institutional Ethical Committee. A written informed consent was obtained from each subject prior to enrolment in the study.
The inclusion criteria ${ }^{26}$ were systemically healthy subjects $>18$ years of age, $>20$ natural teeth. Subjects presenting halitosis of oral origin and an organoleptic score >1 (Rosenberg et al. 1991) were included as cases while no history of halitosis and an organoleptic score 0 as healthy controls.

The exclusion criteria were history of periodontal treatment for the past 1 year, history of any systemic disease, pregnancy and lactation, current smokers, Halitophobic patients, patients under long term antibiotics, immunosuppressants or other immunomodulatory drugs in the past 6 months, habits of masking agents such as mouthwashes / fresh mint / chewing gums / lozenges.

All the study subjects were screened for Oral Hygiene Index, DMFT Index, Probing Pocket Depth (PPD) and Clinical Attachment Level (CAL), Winkel tongue coating index and organoleptic scoring were recorded for all subjects. Organoleptic ratings were obtained only in the morning. For the organoleptic evaluation, participants were instructed to close their mouth for $1 \mathrm{~min}$, and then to slowly exhale air, at a distance of approximately $10 \mathrm{~cm}$ from the nose of the examiner. The odour is scored according to the intensity from 0 to 5.Organoleptic scoring was assessed by single trained examiner. Odour assessment by the examiner was evaluated for 2 consecutive days for 10 patients for calibration. The correlation coefficient of examiner for odor assessment was 0.96 .

Samples collected were supragingival plaque, subgingival plaque and posterior tongue as shown in Fig.1. Pooled samples from 8 sites ( 2 from each quadrant) were collected for supra gingival and subgingival plaque samples. ${ }^{11}$ Supra gingival plaque was removed from the cervical margin of the tooth with a sterile sickle scaler. After the removal of all supra gingival plaque, the subgingival plaque was obtained from the gingival crevice using a sterile curette. Tongue coating was collected with a tongue scrapper from posterior one third of the dorsum of the tongue. The collected samples were stored in Eppendorf tubes with $2 \mathrm{ml}$ of $1 \%$ PBS buffer at $-20^{\circ} \mathrm{C}$ until processing.

DNA extraction was done using commercially available kit (Quiagen ID: 51304. QIAamp DNA Mini Kit, Germany). Quality of the DNA was checked by 0.8\% Agarose gel electrophoresis under UV chamber. A conventional PCR was used to detect the DNA of archaea in the samples. $16 \mathrm{~S}$ ribosomal DNA fragments of oral archaea were amplified using forward and reverse archaeal primers (Biosource, Chennai): SDArch0333aS15 (5'-TCCAGGCCCTACGGG-3') and SDArch0958aA19 (5'-YCCGGCGTTGAMTCCAATT $\left.-3^{\prime}\right)$ targeting the archaeal domain broadly. ${ }^{27}$

The PCR reaction volume consisted of $10 \mu \mathrm{l}$ which included $5 \mu 1$ of PCR mastermix (Genei, Bangalore, 0.4 $\mu 1$ forward and reverse primer, $2.2 \mu l$ of sterile water and $2 \mu \mathrm{l}$ of template DNA.PCR Master mix was composed of Taq DNA polymerase $(0.05 \mathrm{U} / \mu \mathrm{l}), 10 \mathrm{X}$ Taq buffer, 
$4 \mathrm{mM} \mathrm{MgCl}_{2}$ and $0.4 \mathrm{mM}$ dNTP. The amplification was done in the PCR thermocycler as mentioned in table 1. PCR was analyzed using $2 \%$ Agarose gel electrophoresis. The gel was run at $100 \mathrm{mV}$ for 20 minutes and then visualized in UV trans-illuminator and imaged. The presence of sharp bands were analyzed and noted positive for the presence of archaea as shown in Fig. 2.

\section{Statistical Analysis}

The collected data were analysed with IBM.SPSS statistics software 23.0 Version. To find the significant difference between the bivariate samples in Paired groups, the Paired sample t-test was used \& for Independent groups, the unpaired sample t-test was used. Chi-Square test and Fisher's Exact was used to find the significance in categorical variable; Mc Nemar test was used to find the significant difference between dichotomous dependent variable between related groups. In all the above statistical tools the probability value .05 is considered as significant level.

\section{Results}

The demographic data, OHI score, plaque score, WTCI, probing depth and Organoleptic scoring of the study population is summarized in Table 2. Both groups had a similar age, gender distribution, OHI, probing depth while a statistically significant difference was observed in the Tongue coating index and organoleptic scoring between the cases and controls. Out of 50 patients included, 18 were healthy gingiva, 26 were gingivitis and 6 were periodontitis. The mean OHI (S) score, plaque score, WTCI, probing depth and Organoleptic scoring in Table 2.
Fig. 3 shows graphical representation of prevalence of methanogenic archaea in the study groups. Table 3 shows the prevalence of methanogenic archaea in supra gingival plaque, subgingival plaque and tongue coating in halitosis subjects were $16 \%, 60 \%$ and $16 \%$ respectively and in controls without halitosis were $4 \%$, $8 \%$ and $0 \%$ respectively.

Intergroup comparisons (Table 4) of distribution of methanogenic archaea in supra gingival plaque, subgingival plaque and tongue coating, revealed a highly statistical significant difference between prevalence of archaea in subgingival plaque samples of cases and controls. However, there was no statistically significant difference between prevalence of methanogenic archaea in supra gingival and tongue coating of cases and controls.

Within the halitosis group (Table 5), comparing for distribution of methanogenic archaea between supra gingival plaque, sub gingival plaque and tongue coating showed a statistically significant difference between supra gingival plaque $\&$ subgingival plaque and also in subgingival plaque \& tongue coating. However, there was no statistically significant difference between, supra gingival plaque and tongue coating samples. When intra group comparisons were made within controls (Table 5), comparing for distribution of methanogenic archaea between supra gingival plaque, subgingival plaque and tongue there was no statistically significant difference between either of them.

In addition, an intra group correlation was made for OLS and WTCI with respect to presence of methanogenic archaea in cases (Table 6), there was a significant correlation between OLS and presence of methanogenic archaea.

Table 1: Program for PCR

\begin{tabular}{|l|c|c|c|c|}
\hline \multicolumn{1}{|c|}{ Denaturation } & Annealing & Extension & Final Extension & Hold \\
\hline $94^{\circ} \mathrm{C}$ & $60^{\circ} \mathrm{C}$ & $72^{\circ} \mathrm{C}$ & $72^{\circ} \mathrm{C}$ & $4^{\circ} \mathrm{C}$ \\
\hline $30 \mathrm{~s}$ & $30 \mathrm{~s}$ & $40 \mathrm{~s}$ & $7 \mathrm{~m}$ & $\infty$ \\
\hline 35 cycles & & & \\
\hline
\end{tabular}

Table 2: The mean and standard deviation of the demographic data of the study groups

\begin{tabular}{|l|c|c|c|}
\hline \multicolumn{1}{|c|}{ Parameters } & Cases & Controls & P Value \\
\hline Age (years) & $30 \pm 10.29$ & $29 \pm 10.14$ & 0.836 \\
\hline Gender (M/F) & $16 / 9$ & $13 / 12$ & 0.39 \\
\hline OHI-S & $3.67 \pm 1.05$ & $3.54 \pm 1.15$ & 0.684 \\
\hline PPD $(\mathrm{mm})$ & $2.40 \pm 0.58$ & $2.42 \pm 0.55$ & 0.941 \\
\hline Plaque Index & $2.08 \pm 0.39$ & $2.13 \pm 0.35$ & 0.683 \\
\hline WTCI & $7.8 \pm 2.23$ & $5.56 \pm 0.00$ & $0.004^{* *}$ \\
\hline OLS & $4.32 \pm 0.69$ & $0.00 \pm 0.00$ & $0.000^{* *}$ \\
\hline
\end{tabular}

$* \mathrm{p}<0.05$ was considered statistically significant

**Highly significant 
Table 3: Prevalence of methanogenic archaea in supra gingival plaque, subgingival plaque and tongue coating of study groups

\begin{tabular}{|l|c|c|c|c|}
\hline Groups & Samples & Positive & Negative & Prevalence (\%) \\
\hline Cases & Supra gingival plaque & 4 & 21 & 16 \\
\cline { 2 - 5 } & Subgingival plaque & 15 & 10 & 60 \\
\cline { 2 - 5 } & Tongue coating & 4 & 21 & 16 \\
\hline \multirow{3}{*}{ Controls } & Supra gingival plaque & 1 & 24 & 4 \\
\cline { 2 - 5 } & Subgingival plaque & 2 & 23 & 8 \\
\cline { 2 - 5 } & Tongue coating & 0 & 25 & 0 \\
\hline
\end{tabular}

Table 4: Inter group comparisons for presence of methanogenic archaea in cases and controls using Fisher's exact test

\begin{tabular}{|l|c|c|c|}
\hline \multicolumn{1}{|c|}{ Positive } & $\begin{array}{c}\text { Cases } \\
\mathbf{n}(\boldsymbol{\%})\end{array}$ & $\begin{array}{c}\text { Controls } \\
\mathbf{n}(\boldsymbol{\%})\end{array}$ & P value \\
\hline Supra gingival & $4(16)$ & $1(4)$ & 0.359 \\
\hline Sub gingival & $15(60)$ & $2(8)$ & $0.000^{* *}$ \\
\hline Tongue coating & $4(16)$ & $0(0)$ & 0.110 \\
\hline
\end{tabular}

$* p<0.05$ was considered statistically significant;

**Highly significant

Table 5: Intra group comparison for presence of methanogenic archaea in cases and controls using McNemar test

\begin{tabular}{|l|c|c|}
\hline & Cases & Controls \\
\hline Supra gingival \& supragingival & $0.001^{* *}$ & 1.00 \\
\hline Supragingival \& tongue coating & 1.00 & 1.00 \\
\hline Supragingival \& tongue coating & $0.001^{* *}$ & 0.5 \\
\hline
\end{tabular}

$* \mathrm{p}<0.05$ was considered statistically significant

***Highly significant

Table 6: Intra group correlation between clinical parameters and presence of methanogenic archaea using unpaired $t$ test

\begin{tabular}{|l|c|c|c|}
\hline Parameter & Mean \& SD & Positive cases (n) & P value \\
\hline OLS & $4.32 \pm 0.69$ & 15 & $0.001^{* *}$ \\
\hline WTCI & $7.8 \pm 2.23$ & 15 & 0.280 \\
\hline
\end{tabular}

$* \mathrm{p}<0.05$ was considered statistically significant

**Highly significant

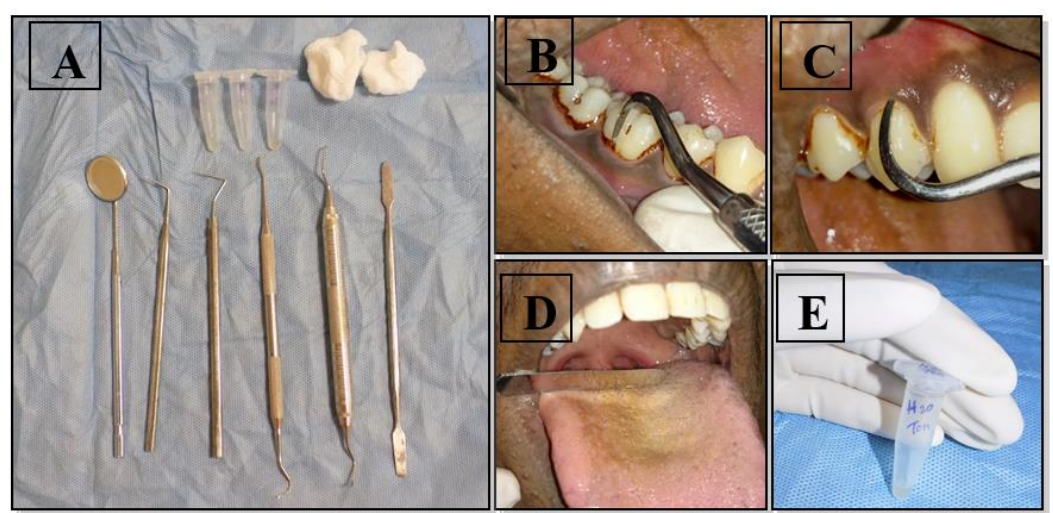

Fig. 1: Sample collection; A: Armamentarium; B: Supra gingival plaque; C: Subgingival plaque; D: Tongue coating; E: Collected sample 


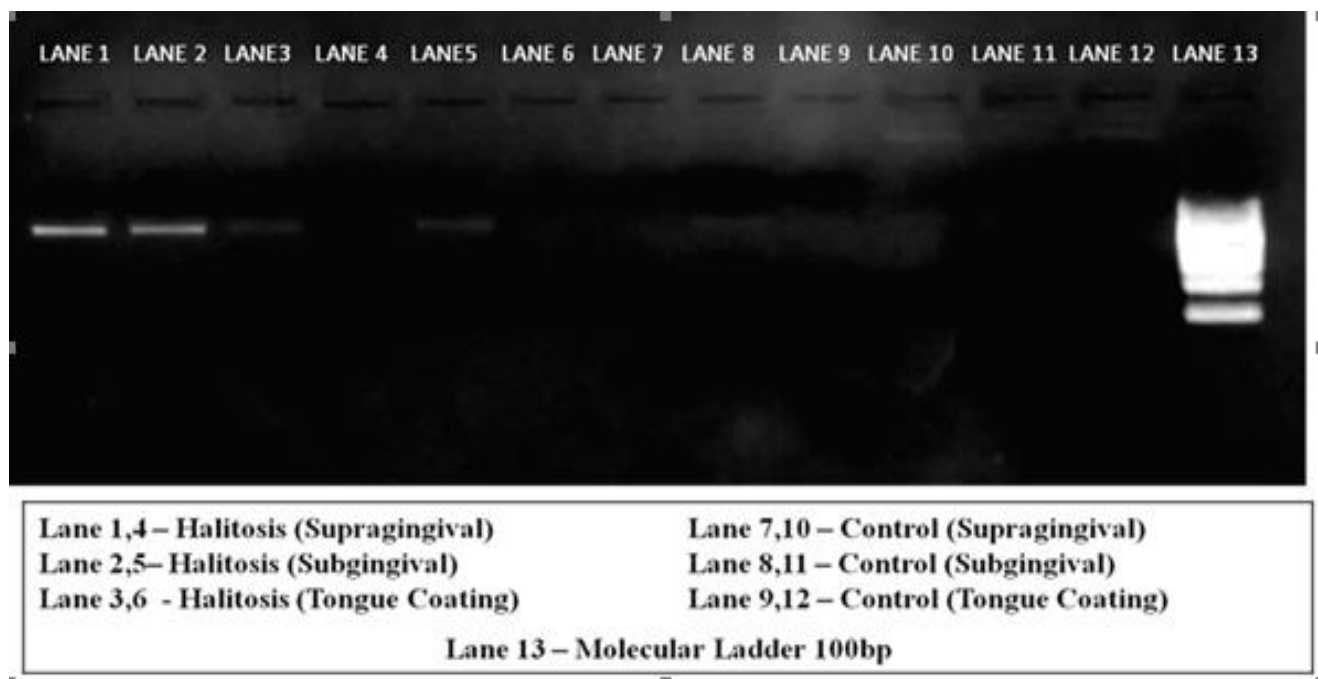

Fig. 2: Methanogenic archaea visualized in UV transilluminator as sharp radiopaque bands

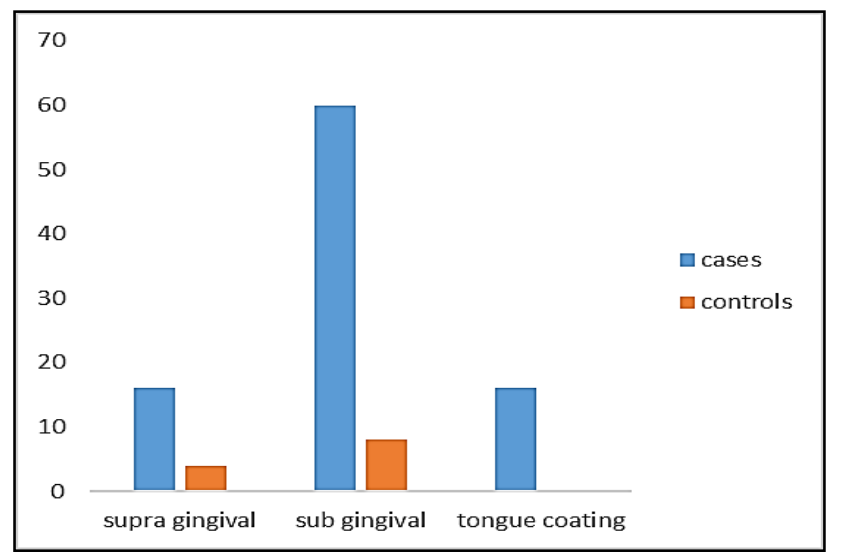

Fig. 3: Graphical representation of distribution of methanogenic archaea in study groups

\section{Discussion}

The present study evaluated the prevalence of methanogenic archaea in subjects with and without halitosis. Methanogenic archaea was detected in supra gingival plaque and subgingival plaque of both the groups whereas with respect to tongue coating, the archaea was present in the halitosis group and absent in the control group. There was a significantly higher presence of methanogenic archaea in the subgingival plaque samples of halitosis patients compared to controls.

Halitosis is the term used to describe any disagreeable odor in the breath. Prevalence of halitosis ranges around $15-78 \%$ and amongst $>20$ years of age with a 3 times higher male predilection. ${ }^{28,29)}$ Persistent halitosis occurs as a result of intraoral causes that usually originates from the posterior dorsum of the tongue and/or oral diseases. ${ }^{15}$ Studies provide evidence that oral microflora produces VSC and organic acids causing halitosis. ${ }^{30}$

Periodontal pathogens Porphyromonas gingivalis, Prevotella intermedia, Tannerella forsythia, Treponema denticola,species of bacteriodes, spirochetes, candida, actinomyces have been associated with production of VSC and thereby implicated in oral halitosis. ${ }^{18-20,31,32}$ However oral halitosis have also been identified in subjects without periodontal disease wherein other microflora may play a role. The present study was performed to identify the distribution and prevalence of methanogenic archaea amongst patients with halitosis.

Methods of halitosis assessment includes organoleptic scoring, gas chromatography, Halimeter, Oral Chroma, BANA test, salivary incubation test, etc. Of the above mentioned methods, gas chromatography, halimeter, oral chroma, BANA are designed to detect VSC. Halimeter detects intra-oral halitosis while Oral Chroma, gas chromatography can perfectly differentiate between intra-oral and extra-oral blood-borne halitosis. ${ }^{33}$ Kursun et al in 2013 showed a significant correlation between organoleptic assessment and halimeter. ${ }^{34}$ OLS has the highest correlation to detect slight to severe halitosis. Due to high cost of these equipment and OLS being the gold standard, ${ }^{35}$ in our study we have used OLS as diagnostic criteria for selecting subjects with halitosis. All patients included in the study had a score $>/=3$. The use of OLS as a case definition criteria was further 
validated by the observation of significant correlation between presence of methanogenic archaea and OLS in the halitosis group. $\mathrm{p}=0.001$

Dorsum of the tongue is the primary source of VSC causing oral malodor both in periodontally diseased and healthy subjects. ${ }^{15}$ Gram negative facultative bacteria have been isolated on tongue dorsa which contributed significantly to VSC production. ${ }^{19}$ Studies reveal that daily brushing or scraping of tongue reduces the substrata for putrefaction. ${ }^{36}$ In the present study, there was a significantly higher presence of tongue coating in the halitosis group than control. Methanogenic archaea was detectable in $16 \%$ of tongue coating samples from halitosis group, whereas it was notably absent in tongue coating samples from control group.

Methanogens are strict anaerobes characterized by the ability to produce methane from $\mathrm{CO}_{2}$ and $\mathrm{H}_{2}$ and in some cases from formate, acetate or methanol. Hydrogen is the waste end product of the metabolism of microorganisms in anoxic environment. Maintaining a low hydrogen concentration is necessary for anaerobic fermentative process. Methanogens depend on hydrogen and carbon dioxide produced by other species; in return some species grow better in the presence of the methanogens because of the altered patterns of the redox balance associated with reduced partial pressure of hydrogen due to interspecies hydrogen transfer. ${ }^{37}$ Methanobrevibacter, the major genus of methanogenic archaea are anaerobes, isolated from the oral cavity and pocket depth provides an anaerobic environment that is required for their colonization. ${ }^{10}$ It has been postulated that syntrophy occurs in the anaerobic microbial community of deep periodontal pockets, where methanogens consume $\mathrm{H}_{2}$ produced by secondary fermenters, and contributes to periodontal disease. ${ }^{38}$

Methanogenesis is performed by anaerobic consortia that degrade biological compounds including lipids, carbohydrates and proteins. ${ }^{39}$ From the system biology view point, the quantity and the methane production by archaea is more likely a reflection of syntrophic relationships in the gut where the local environment, depending on a number of factors, may favor hydrogen channeling through alternative mechanisms of hydrogen disposal, such as methanogenesis, sulfate reduction, or acetogenesis. ${ }^{40}$ Alternative generation of highly toxic hydrogen sulfide as a result of sulfate reduction in the gut may impose much higher health risks compared to more inert methane. ${ }^{41}$ Similar generation of hydrogen sulfide and methane has been proposed as a possible mechanism of VSC production causing intra oral halitosis. In accordance to this, our study assessed the prevalence of methanogenic archaea in halitosis subjects.

Literature on the prevalence of methanogenic archaea in plaque samples of subjects with healthy gingiva and periodontitis have given conflicting results. ${ }^{27,42,43}$ Methanogenic archaea was also assessed in tongue coating and subgingival plaque of healthy and periodontitis patients but was predominantly seen in subgingival plaque samples of healthy and periodontitis.,10,42 Based on the above literature sampling has been done in the present study by pooling supra gingival plaque and subgingival plaque samples from 8 sites ( 2 from each quadrant selected in random) and single sample from posterior third of tongue dorsum in each subject of both the groups.

Methanogenic archaea have been implicated in several oral inflammatory conditions including periodontitis, inflamed pulp tissue and periimplantitis. ${ }^{7,84}$ Lepp et al identified methanogenic archaeal DNA from $36 \%$ of subgingival plaques of periodontitis patients. $^{42} \mathrm{Li}$ et al showed that methanogenic archaea were found in subgingival plaque of $65 \%$ of aggressive periodontitis patients, $72 \%$ of chronic periodontitis, $26 \%$ of gingivitis and a complete absence in healthy subjects suggesting that methanogenic archaea may be implicated as causative agent for periodontitis. ${ }^{43}$ However, a lower prevalence of methanogenic archaeal detection with $11.8 \%$ of subgingival plaque in healthy controls and $29.4 \%$ of subgingival plaque in chronic periodontitis patients was reported by Ashok et al. ${ }^{27}$ Matarazzo et al have also detected archaea in subgingival plaque of $68 \%$ and $58.3 \%$ of sites with generalized aggressive periodontitis and periodontally healthy gingival sulcus respectively. ${ }^{4}$ Although the present study was carried out using case definition criteria for halitosis, out of the total of 50 subjects included in the study, 18 had healthy gingiva, 26 had chronic gingivitis and 6 were identified to be having chronic periodontitis. Our results showed methanogenic archaea to be present in $38 \%$ of subgingival plaque of healthy gingiva, $30 \%$ in subgingival plaque of chronic gingivitis and 33\% of subgingival plaque of chronic periodontitis patients. It is also of interest to note that methanogenic archaea was identified in samples from $60 \%$ of subjects belonging to the halitosis group, whereas methanogenic archaeal presence was observed only in $8 \%$ of the samples from the control group independent of periodontal status.

To the best of our knowledge, our study is the first to identify and assess the prevalence of methanogenic archaea in halitosis subjects, revealing a significantly higher presence in the halitosis subjects $(60 \%)$ than controls. The predominance of methanogenic archaea in the subgingival sites can be explained by is characteristic of being an obligate anaerobe.

Methanogenic archaea may not be the only source of VSC production, however, it may be one of the important organism contributing to its production due to synergistic or syntrophic relationship with other microbes. Further studies with large sample size, estimating VSC levels and archaeal quantification are needed to understand methanogenic archaea as a potential pathogen for halitosis. 


\section{Conclusion}

Methanogenic archaea are single celled methanogenic anaerobic microorganisms that are potential hydrogen competitors. It serves as a syntrophic partners with other members of the subgingival biofilm community leading to methanogenesis, sulfate reduction and acetogenesis that are associated to produce hydrogen sulfide, a potent VSC to cause halitosis.

A significantly higher presence of methanogenic archaea was observed in halitosis subjects than controls, with increased detection in the subgingival plaque compared to supra gingival plaque and tongue coating. The preliminary results of our study fulfill the association criteria of Socransky's postulates. However, a larger sample size, identification of VSC production and synergistic activity with other microorganisms needs to be established.

\section{Conflict of Interest: None.}

\section{References}

1. Woese CR, Kandler O, Wheelis ML. Towards a natural system of organisms: proposal for the domains Archaea, Bacteria, and Eucarya. Proc Natl Acad Sci. 1990;87:4576-9.

2. Kandler O, König H. Cell wall polymers in Archaea (Archaebacteria). Cell Mol Life Sci. 1998;54:305-8.

3. Graham DE, Overbeek R, Olsen GJ, Woese CR. An archaeal genomic signature. Proc Natl Acad Sci. 2000;97:3304-8.

4. Matarazzo F, Ribeiro AC, Feres M, Faveri M, Mayer MP. Diversity and quantitative analysis of Archaea in aggressive periodontitis and periodontally healthy subjects. J Clin Periodontol. 2011;38:621-7.

5. Belay N, Mukhopadhyay B, De Macario EC, Galask R, Daniels L. Methanogenic bacteria in human vaginal samples. J Clin Microbiol. 1990;28:1666-8.

6. Kulik EM, Sandmeier H, Hinni K, Meyer J. Identification of archaeal rDNA from subgingival dental plaque by PCR amplification and sequence analysis. FEMS Microbiology Letters. 2001;196:129-33.

7. Vickerman MM, Brossard KA, Funk DB, Jesionowski AM, Gill SR. Phylogenetic analysis of bacterial and archaeal species in symptomatic and asymptomatic endodontic infections. J Med Microbiol. 2007;56:110-8.

8. Faveri M, Gonçalves LF, Feres M, Figueiredo LC, Gouveia LA, Shibli JA et al. Prevalence and microbiological diversity of Archaea in peri-implantitis subjects by $16 \mathrm{~S}$ ribosomal RNA clonal analysis. $J$ Periodontal Res. 2011;46:338-44.

9. Li CL, Liu DL, Jiang YT, Zhou YB, Zhang MZ, Jiang W et al. Prevalence and molecular diversity of Archaea in subgingival pockets of periodontitis patients. Oral Microbiol Immunol. 2009;24:343-6.

10. Robichaux M, Howell M, Boopathy R. Growth and activities of sulfate-reducing and methanogenic bacteria in human oral cavity. Current Microbiol. 2003;47:12-6.

11. Vianna ME, Holtgraewe S, Seyfarth I, Conrads G, Horz HP. Quantitative analysis of three hydrogenotrophic microbial groups, methanogenic archaea, sulfate-reducing bacteria, and acetogenic bacteria, within plaque biofilms associated with human periodontal disease. J bacterial. 2008;190:3779-85.
12. De Boever EH, Loesche WJ. Assessing the contribution of anaerobic microflora of the tongue to oral malodor. $J$ Am Dent Assoc. 1995;126:1384-93.

13. Porter SR, Scully C. Oral malodour (halitosis). BMJ. 2006;333:632-5.

14. Delanghe G, Ghyselen J, van Steenberghe D, Feenstra L. Multidisciplinary breath-odour clinic. Lancet. 1997;350:187.

15. Yaegaki K, Sanada K. Volatile sulfur compounds in mouth air from clinically healthy subjects and patients with periodontal disease. J Periodontal Res. 1992;27:2338 .

16. Tonzetich J, Coil JM, Ng W. Gas chromatographic method for trapping and detection of volatile organic compounds from human mouth air. J Clin Dent. 1991;2:79-82.

17. Persson S, Edlund MB, Claesson R, Carlsson J. The formation of hydrogen sulfide and methyl mercaptan by oral bacteria. Oral Microbiol Immunol. 1990;5:195-201.

18. Zhu WD, Sha YQ, Chen ZB. Effect of spirochetes and porphyromonus gingivalis on oral malodor. Chin $J$ Stomatol. 2003;38:206-9.

19. Tanaka M, Yamamoto Y, Kuboniwa M, Nonaka A, Nishida N, Maeda K et al. Contribution of periodontal pathogens on tongue dorsa analyzed with real-time PCR to oral malodor. Microbes Infect. 2004;6:1078-83.

20. Washio J, Sato T, Koseki T, Takahashi N. Hydrogen sulfide-producing bacteria in tongue biofilm and their relationship with oral malodour. J Med Microbiol. 2005;54:889-95.

21. Tangerman A, Winkel EG. Intra-and extra-oral halitosis: finding of a new form of extra-oral blood-borne halitosis caused by dimethyl sulphide. J Clin Periodontol. 2007;34:748-55.

22. Goldberg S, Kozlovsky A, Gordon D, Gelernter I, Sintov A, Rosenberg M. Cadaverine as a putative component of oral malodor. J Dent Res. 1994;73:1168-72.

23. Yoneda M, Masuo Y, Suzuki N, Iwamoto T, Hirofuji T. Relationship between the $\beta$-galactosidase activity in saliva and parameters associated with oral malodor. $J$ Breath Res. 2010;4: 017108.

24. Boopathy R, Robichaux M, LaFont D, Howell M. Activity of sulfate-reducing bacteria in human periodontal pocket. Can J Microbiol. 2002;48:1099-103.

25. Tonzetich J. Production and Origin of Oral Malodor: A Review of Mechanisms and Methods of Analysis. $J$ Periodontol. 1997;48:13-20.

26. Winkel EG, Roldan S, Van Winkelhoff AJ, Herrera D, Sanz M. Clinical effects of a new mouthrinse containing chlorhexidine, cetylpyridinium chloride and zinc-lactate on oral halitosis. J Clin Periodontol. 2003;30:300-6.

27. Ashok N, Warad S, Singh VP, Chaudhari H, Narayanan A, Rodrigues J. Prevalence of archaea in chronic periodontitis patients in an Indian population. Indian $J$ Dent Res. 2013;24:289-93.

28. Nadanovsky PA, Carvalho LB, Ponce de Leon A. Oral malodour and its association with age and sex in a general population in Brazil. Oral Dis. 2007;13:105-9.

29. Hammad MM, Darwazeh AM, Al-Waeli H, Tarakji B, Alhadithy TT. Prevalence and awareness of halitosis in a sample of Jordanian population. J Int Soc Prev Community Dent. 2014;4:178-86.

30. Tonzetich J, Richter V. Evaluation of volatile odoriferous components of saliva. Arch Oral Biol. 1964;9:39-45.

31. Awano S, Gohara K, Kurihara E, Ansai T, Takehara T. The relationship between the presence of periodontopathogenic bacteria in saliva and halitosis. Int Dental J. 2002;52:212-6. 
32. Koga C, Yoneda M, Nakayama K, Yokoue S, Haraga M, Oie $\mathrm{T}$ et al. The detection of Candida species in patients with halitosis. Int J Dent. 2014;857647.

33. Tangerman A, Winkel EG. The portable gas chromatograph OralChroma ${ }^{\mathrm{TM}}$ : a method of choice to detect oral and extra-oral halitosis. J Breath Res. 2008;2:017010.

34. Kursun S, Acar B, Atakan C, Öztas B, Paksoy CS. Relationship between genuine and pseudohalitosis and social anxiety disorder. J Oral Rehabil. 2014;41:822-8.

35. Erovic Ademovski S, Lingström P, Winkel E, Tangerman A, Persson GR, Renvert S. Comparison of different treatment modalities for oral halitosis. Acta Odontol Scand. 2012;70:224-33.

36. Bollen CM, Beikler T. Halitosis: the multidisciplinary approach. Int J Oral Sci. 2012;4:55-63.

37. Jiang YT, Xia WW, Li CL, Jiang W, Liang JP Preliminary study of the presence and association of bacteria and archaea in teeth with apical periodontitis. Int Endod J. 2009;42:1096-103.

38. Eckburg PB, Lepp PW, Relman DA. Archaea and their potential role in human disease. Infect Immun. 2003;71:591-6.

39. Alves MM, Pereira MA, Sousa DZ, Cavaleiro AJ, Picavet M, Smidt $\mathrm{H}$ et al. Waste lipids to energy: how to optimize methane production from long-chain fatty acids (LCFA). Microb Biotechnol. 2009;2:538-50.

40. Matarazzo F, Ribeiro AC, Faveri M, Taddei C, Martinez MB, Mayer MP. The domain Archaea in human mucosal surfaces. Clinical Microbiol Infect. 2012;18:834-40.

41. Carbonero F, Benefiel AC, Alizadeh-Ghamsari AH, Gaskins HR. Microbial pathways in colonic sulfur metabolism and links with health and disease. Front Physiol. 2012;3:00448.

42. Lepp PW, Brinig MM, Ouverney CC, Palm K, Armitage GC, Relman DA. Methanogenic Archaea and human periodontal disease. Proc Natl Acad Sci. 2004;101:617681.

43. Li, Chao Lun. Prevalence and quantification of the uncommon Archaea phylotype Thermoplasmata in chronic periodontitis. Arch Oral Biol. 2014;59:822-8.

How to cite this article: Ayswarya V, Lavu V, Rao S. R. Distribution of methanogenic archaea in different oral sites amongst subjects with halitosis. Indian J Microbiol Res. 2018;5(4):516-523. 